\title{
HOBYS and W43-HERO: \\ Two more steps toward a Galaxy-wide understanding of high-mass star formation
}

\author{
Frédérique Motte ${ }^{1,2}$, Sylvain Bontemps ${ }^{3}$ and Jérémy Tigé ${ }^{4}$ \\ ${ }^{1}$ Institut de Planétologie et d'Astrophysique de Grenoble (IPAG), \\ Univ. Grenoble Alpes - CNRS/INSU, BP 53, 38041 Grenoble Cedex 9, France \\ email: frederique.motte@ujf-grenoble.fr \\ ${ }^{2}$ Laboratoire AIM Paris-Saclay, CEA/IRFU - CNRS/INSU - Univ. Paris Diderot, \\ Service d'Astrophysique, Bât. 709, CEA-Saclay, F-91191 Gif-sur-Yvette Cedex, France \\ ${ }^{3}$ OASU/LAB-UMR 5804, CNRS - Université Bordeaux 1, 33270 Floirac, France \\ ${ }^{4}$ Laboratoire dAstrophysique de Marseille (LAM), Aix Marseille Université - CNRS, \\ UMR 7326, 13388, Marseille, France
}

\begin{abstract}
The Herschel/HOBYS key program allows to statistically study the formation of $10-20 M_{\odot}$ stars. The IRAM/W43-HERO large program is itself dedicated to the much more extreme W43 molecular complex, which forms stars up to $50 M_{\odot}$. Both reveal high-density cloud filaments of several $\mathrm{pc}^{3}$, which are forming clusters of OB-type stars. Given their activity, these so-called mini-starburst cloud ridges could be seen as "miniature and instant models" of starburst galaxies. Both surveys also strongly suggest that high-mass prestellar cores do not exist, in agreement with the dynamical formation of cloud ridges. The HOBYS and W43 surveys are necessary steps towards Galaxy-wide studies of high-mass star formation.
\end{abstract}

Keywords. stars: formation, ISM: structure, star clusters, starburst

\section{Introduction}

Despite their low numbers, massive stars (OB-type, $>10 M_{\odot}$ on the main sequence) produce much of the luminosity of galaxies and are the main driver of their evolution. Both their large impact on their environment and how their formation is linked to natal clouds remain poorly understood (see reviews by Elmegreen 2011; Krumholz 2014).

High-mass star formation scenarios currently undergo a change of paradigm, in which this process is no longer quasi-static but simultaneously evolves with both cloud and cluster formation. Theoretically, OB stars could either form through (1) a powerful accretion driven by a high degree of turbulence (e.g., McKee \& Tan 2002; Hosokawa \& Omukai 2009) or (2) colliding flows initiated by competitive accretion or cloud formation (e.g., Bonnell \& Bate 2006; Hartmann et al. 2012). From an observational point of view, gravitational streamers and shearing motions have been reported from the cloud to the protostellar scales in a few high-mass star-forming regions (on $10-0.1$ pc scales, Schneider et al. 2010; Csengeri et al. 2011a). We hereafter show that these pioneering studies are now comforted by sensitive Herschel images, cloud kinematic studies, and (sub)millimeter interferometric images, favoring the second family of models.

"HOBYS, the Herschel imaging survey of OB Young Stellar objects" is a key program, which is exclusively dedicated to high-mass star formation (Motte, Zavago, Bontemps et al. 2010; see http://hobys-herschel.cea.fr). It uses the SPIRE and PACS cameras of the Herschel spatial observatory to image essentially all of the regions forming OB-type stars at distances less than $3 \mathrm{kpc}$ from the Sun $(d=0.7-3.2 \mathrm{kpc})$. HOBYS images 


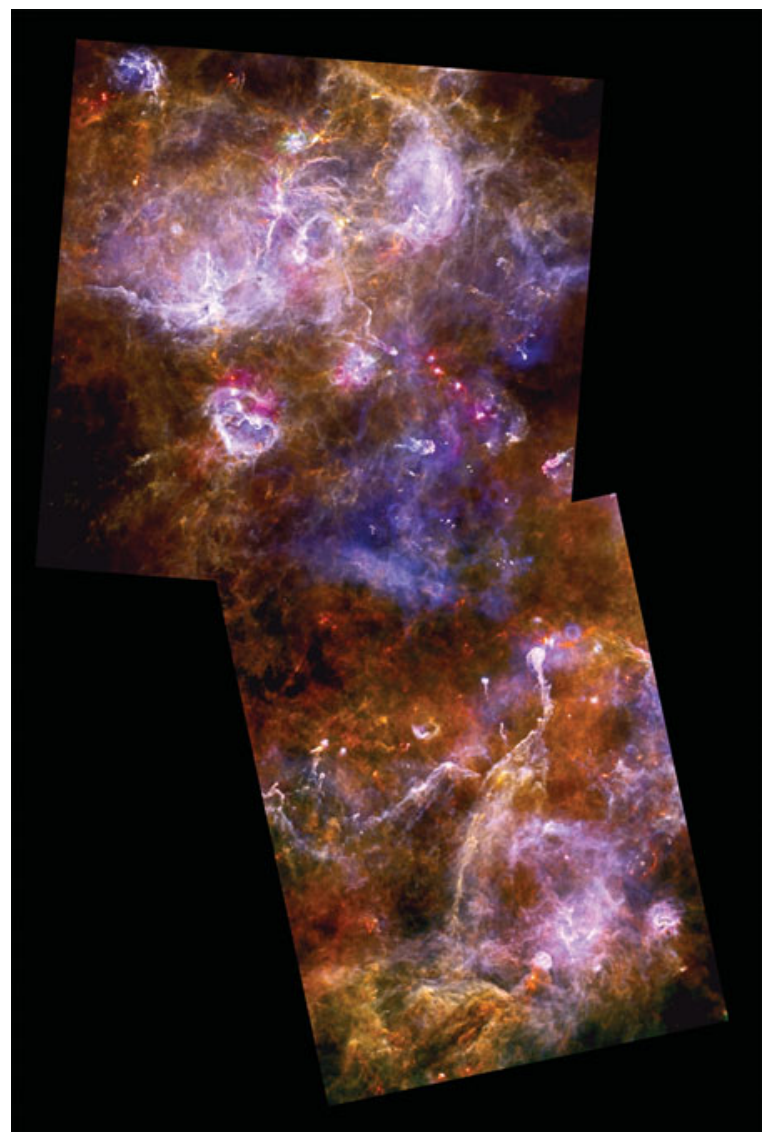

Figure 1. Composite 3-color Herschel image of the Cygnus X molecular complex with red $=250 \mu \mathrm{m}$, green $=160 \mu \mathrm{m}$, and blue $=70 \mu \mathrm{m}$ (taken from Hennemann et al. 2012 and Schneider et al. in prep.). The mosaic performed by the HOBYS key program approximately covers a $5^{\circ} \times 2.5^{\circ}$ or $120 \mathrm{pc} \times 60 \mathrm{pc}$ area. The diffuse blue emission at the center is an $\mathrm{H}$ II region powered by the massive Cyg OB2 cluster while earlier stage star-forming sites are seen as red filaments. (A color version of this figure is available online.)

revealed networks of filaments and clusters of burgeoning YSOs (see Fig. 1). The W43HERO large program is dedicated to the "Origins of molecular clouds and star formation in W43". This extreme molecular complex is located at $5.5 \mathrm{kpc}$ from the Sun, at the tip of the long bar of the Milky Way. This IRAM $30 \mathrm{~m}$ survey is complemented by IRAM Plateau de Bure, Herschel, and ALMA imagings. Among the HOBYS and W43-HERO highlights are the discovery of "mini-starburst ridges" (see Sect. 2) and the recurrent inability to find good prestellar core candidates (see Sect. 3).

\section{Ridges \& hubs: Hyper-massive clumps forming clusters of high-mass stars}

\subsection{Discovery of hyper-massive clumps}

One of the most unexpected results of the Herschel mission was in revealing the importance and ubiquity of interstellar filaments (e.g., André et al. 2010; Hill et al. 2011). They were known from molecular line surveys but could, for the first time, be 1) quantitatively studied thanks to sensitive column density and temperature images and 2) 

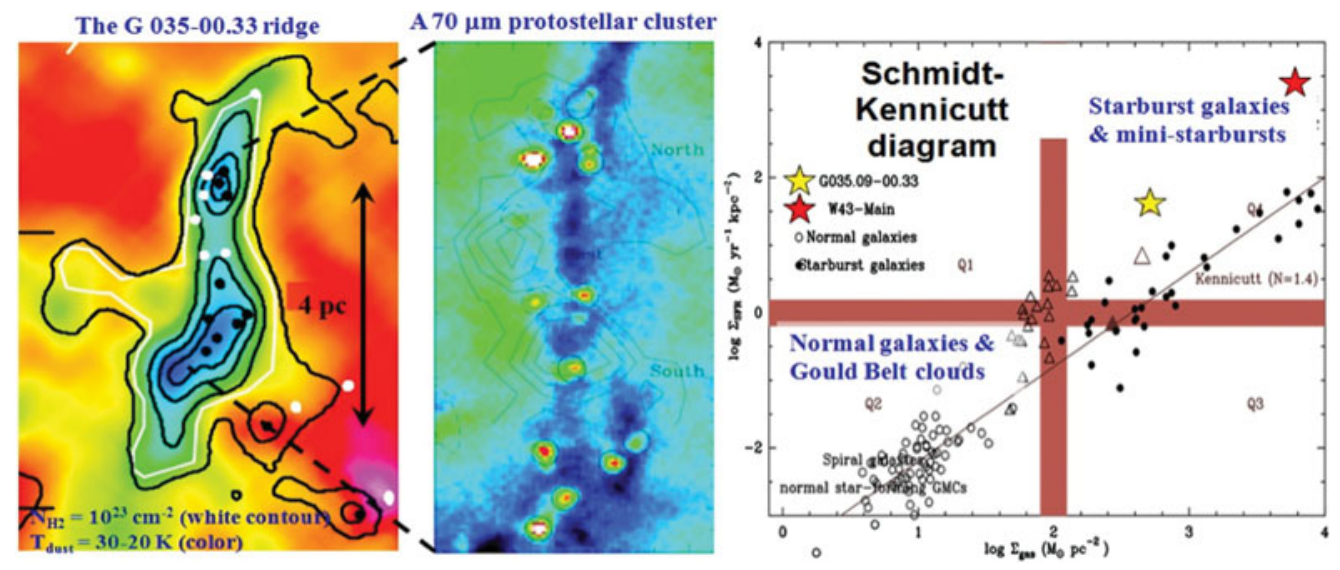

Figure 2. The G035-00.33 ridge and its cluster of high-mass protostars (Left and Center). Ridges are high-density clumps with large star formation activity (Right, see yellow and red star markers). On a Schmidt-Kennicutt diagram, they qualify as mini-starburst regions. Figures extracted from Nguyen Luong et al. (2011a) and (in prep.). (A color version of this figure is available online.)

directly compared with the protostellar population (see, e.g., Figs. 2 Left-Center). The HOBYS group has developed a definite expertise in building clean and reliable column density maps, in identifying and analyzing cloud filaments, in properly extracting protostars from their surroundings and characterizing them (e.g., Hill et al. 2012; Hennemann et al. 2012; Men'shchikov et al. 2012; Tigé et al. 2016). Within the high-mass molecular complexes imaged by HOBYS, we have discovered high-density dominating elongated clumps, which are the preferential sites to form massive stars and which we quote as ridges (see Figs. 2 Left-Center). The existence of ridges is predicted by dynamical models of cloud formation such as colliding flow simulations (e.g., Heitsch \& Hartmann 2008; Federrath et al. 2010). The densest ridges should coincide with the precursors of "young massive clusters" that will become bound stellar clusters (e.g., Ginsburg et al. 2012).

In more details, Hill et al. (2011) studied the Vela C molecular cloud complex and defined the central $\sim 3$ pc-long filament with column density above $10^{23} \mathrm{~cm}^{-2}$ as a ridge. They showed that the Vela $\mathrm{C}$ ridge represents a gravitational well that is dominating and shaping its surroundings, in marked contrast to other more typical filaments (e.g., Arzoumanian et al. 2011). In parallel, Hennemann et al. (2012) analyzed the structure of the DR21 ridge (see Fig. 3 Left), previously identified by us as hosting a cluster of $\sim 20$ high-mass protostars (Motte et al. 2007; Bontemps et al. 2010). In agreement with the kinematical study and simulations of Schneider et al. (2010, see Fig. 3 Right), Hennemann et al. (2012) proposed the DR21 ridge is fed by and formed through the merging of several sub-filaments already forming stars. A handful of other $\sim 10^{4} M_{\odot}$ ridges were recently identified in Herschel/HOBYS and Hi-GAL images (Nguyen Luong et al. 2013; Hill et al. in prep.).

More spherical and less massive, $\sim 10^{3} M_{\odot}$, clumps were also found to form stellar clusters at the junction of filaments (Schneider et al. 2012; Didelon et al. 2015). These clumps recall the so-called "hubs" discovered in a few extreme infrared dark clouds (e.g., Peretto et al. 2013) and predicted by numerical simulations (e.g., Dale \& Bonnell 2011). We propose to generalize the concept of ridges and hubs into that of "hyper-massive clumps", which could either be spherical or filamentary and either form a couple or a rich cluster of high-mass stars. 

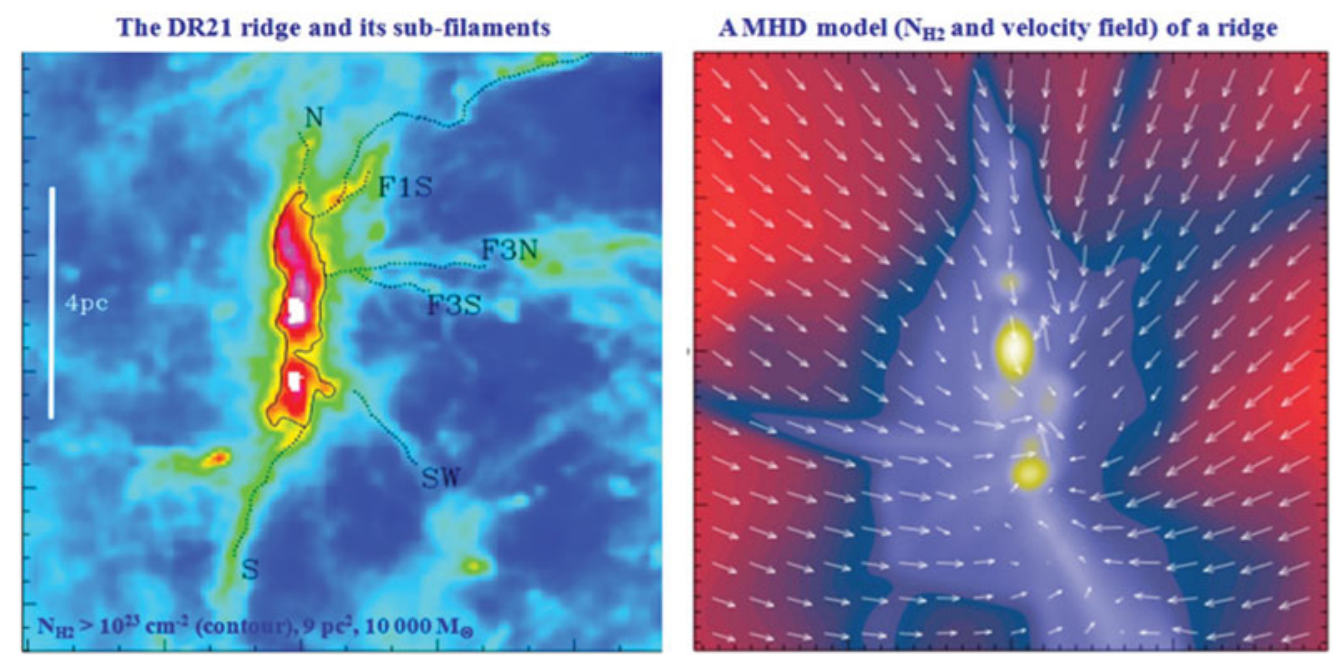

Figure 3. The DR21 ridge and its feeding sub-filaments network (Left), compared to numerical MHD simulations (Right). Panels extracted from Hennemann et al. (2012) and Schneider et al. (2010). (A color version of this figure is available online.)

\subsection{Dynamical processes at the origin of hyper-massive clouds}

Based on Herschel and kinematic studies (Schneider et al. 2010; Hill et al. 2011; Hennemann et al. 2012; Nguyen Luong et al. 2011a, 2013), we proposed that most ridges/hubs could have been formed by dynamical scenarios such as converging flows (see Fig. 3 Right). Minier et al. (2013) themselves used numerical simulations to show that the ionization of a very nearby $\mathrm{H}$ II region/OB cluster could be the main process responsible for shaping some ridges like Vela $\mathrm{C}$ (see his Fig. 5). In any case, whatever the origin of the additional pressure, arising from colliding flows or ionization, it supersedes the thermal and micro-turbulence pressure. Those dynamical scenarios for cloud and then subsequent star formation are part of the second family of models shortly described in Sect. 1. They are also consistent with the observational lack of high-mass prestellar cores (e.g., Motte et al. 2007, 2010; Russeil et al. 2010; Tigé et al. 2016).

In parallel to these Herschel structural studies, we have investigated the kinematics of three ridges identified as such from HOBYS data (Hennemann et al. 2012; Nguyen Luong et al. 2013). A long-term survey of Cygnus X and an IRAM large program on W43 (W43HERO, Motte, Schilke et al., see http://www. iram-institute.org/EN/content-page-2927-158-240-292-0.html) has shown that the DR21, W43-MM1, and W43-MM2 ridges are all undergoing global collapse on the ridge scale with supersonic inward velocities $\left(1-2 \mathrm{~km} \mathrm{~s}^{-1}\right.$ over $1-10 \mathrm{pc}^{2}$; Schneider et al. 2010; Motte et al. in prep.). Such infall motions, sometimes called gravitational focusing, are expected both in colliding flows and ionization compression models (e.g., Vázquez-Semadeni et al. 2005; Tremblin et al. 2012). We started studying the inner kinematic structure of these ridges, which suggests they are braids/bundles of filaments or gas layers (Louvet et al. in prep.; Bontemps et al. in prep.). We have also studied the shocks associated with gas shears created by subfilaments braiding, using $\mathrm{SiO}$ imaging and detailed shock modeling (Nguyen Luong et al. 2013; Duarte-Cabral et al. 2014; Louvet et al. 2016). Such extreme kinematics is observed by other groups at the clump scale (e.g. Galván-Madrid et al. 2010; Henshaw et al. 2014) and is reminiscent of gas flows and shocks observed at the protostellar scale by Csengeri et al. (2011a-b). 


\subsection{Galactic mini-starburst events}

Extreme characteristics of ridges and hubs, in terms of density and kinematics, could lead to an atypical star formation activity. Star formation efficiency is indeed predicted to increase with gas density (Krumholz \& McKee 2005; Hennebelle \& Chabrier 2011; Padoan $\&$ Nordlund 2011). The clear accumulation of massive protostars we observed along ridges (see, e.g., Fig. 2 Center) leads, on $1-10 \mathrm{pc}^{2}$ areas, to star formation rate densities worthy of starburst galaxies (see Fig. 2 Right). For this reason, W43-main, G035.39-00.33, and W43-MM1 qualify as mini-starburst regions, i.e. "miniature and instantaneous models of starburst galaxies" (Motte et al. 2003; Nguyen Luong 2011a; Louvet et al. 2014).

These mini-starburst events most probably follow the formation of the ridges, which were proposed to develop through colliding flows. Indeed, short mini-bursts of star formation are to be expected after a fast episode of cloud formation (e.g., Vázquez-Semadeni et al. 2008) or, equivalently, for a cloud under compressive turbulent forcing (Federrath \& Klessen 2012). In this scenario, star formation should gradually settle within ridges. We should thus measure very different SFR levels depending on the evolutionary status of the ridge. Louvet et al. (2014) measured SFRs within subregions of the W43-MM1 ridge and found a clear correlation of SFR with cloud density or, equivalently, SFR with the evolution of cloud concentration. Moreover, SFR is smaller within the eastern part of the ridge, where the shocks associated with more recent cloud formation are the strongest (Louvet et al. 2014, 2016). Therefore, the dynamical ridge formation may well be followed by series of intense bursts of star formation.

\section{A ten years-long quest for high-mass prestellar cores}

The dynamical picture presented above is consistent with the fact that observers have tried for ten years, in vain, to identify good candidate high-mass prestellar cores (Motte et al. 2007, 2010; Russeil et al. 2010; Tigé et al. 2016). The quest for high-mass prestellar cores started with ground-based millimeter images and continued with sets of Herschel images at far-infrared to submillimeter wavelengths. Given the $1-3 \mathrm{kpc}$ distance of the nearest high-mass star-forming complexes, both surveys are unable to resolve individual high-mass prestellar cores, assumed to be individual mass reservoirs of $\sim 0.02 \mathrm{pc}$ sizes and $>10^{6} \mathrm{~cm}^{-3}$ densities. They however do resolve their parental massive dense cores (MDCs, a few $0.1 \mathrm{pc}$ ). While millimeter surveys failed to find starless MDCs (Motte et al. 2007, Russeil et al. 2010), Herschel studies, thanks to their careful dust temperature measurements, did identify tens of starless MDC candidates (Tigé et al. 2016).

However, several follow-ups do suggest that most starless MDCs may not contain fragments dense and massive enough to be high-mass prestellar cores. A handful of the NGC 6334 starless MDCs, observed at higher-resolution proved indeed not to be centrally concentrated but to mostly contain filaments and diffuse gas (Tigé et al. 2016). Similarly, the starless MDC candidates of the W43-MM1 ridge observed with ALMA disperse into diffuse structures and a few low-mass cores at the $0.02 \mathrm{pc}$ scale (project Motte et al.). When located in the mass versus radius diagram of Fig. 4, starless MDCs are expected to follow the $M(<r) \propto r^{2}$ relation, which links a sample of starless clumps and their prestellar sub-fragments/cores (Butler \& Tan 2012; Tan et al. 2013). This mass concentration is much lower than the one generally found for protostellar envelopes and ridges, which have $\rho \propto r^{-2}$ density structures (e.g., Motte \& André 2001; Didelon et al. $2015)$ and thus $M(<r) \propto r$ mass concentration. At 0.02 pc scales, NGC 6334 starless MDCs of Tigé et al. (2016) and all starless fragments found by Tan et al. (2013), including C1-S, should merely host low- to intermediate-mass prestellar cores. 


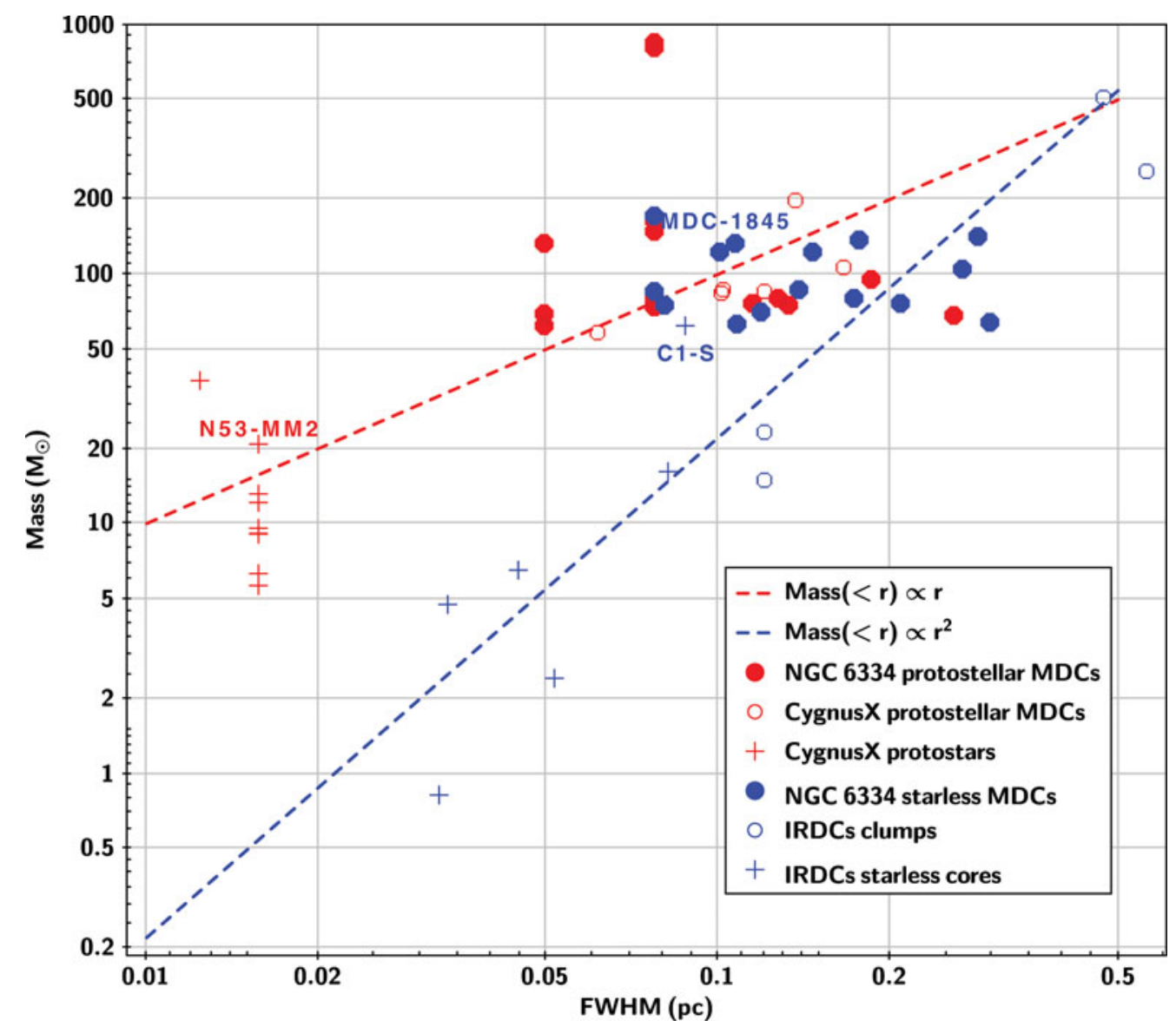

Figure 4. Prediction of gas concentration within starless MDC candidates of NGC 6334 (filled blue circles). Given that they are expected to follow the $M(<r) \propto r^{2}$ relation (yellow dashed line), linking other starless clumps and their prestellar sub-fragments/cores (open blue symbols), NGC 6334 starless MDCs should merely host low- to intermediate-mass prestellar cores. Figure extracted from Tigé et al. (2016). (A color version is available online.)

Pre-stellar cores could also be hosted within IR-quiet (young) protostellar MDCs. An interferometric study of similar objects in Cygnus X however showed that most if not all massive sub-fragments at $0.02 \mathrm{pc}$ scales are associated with protostars (Duarte-Cabral et al. 2013).

After ten years of research, we therefore still lack a good candidate high-mass prestellar core. Several interferometric studies toward large samples of MDCs are ongoing. We are then at the dawn of finally proving that high-mass prestellar cores do or do not exist.

\section{Conclusion}

The dynamical picture of Sect. 2 and the lack of high-mass prestellar cores presented in Sect. 3 are consistent. Indeed, the high-mass equivalent of prestellar cores may be replaced by low-mass protostars within hyper-massive clumps. The latter may thus constitute the pc-scale "gas reservoir", from which gas is accreted onto 0.02 pc-scale protostellar envelopes and in turn onto protostellar embryos. As a consequence, protostars will grow 

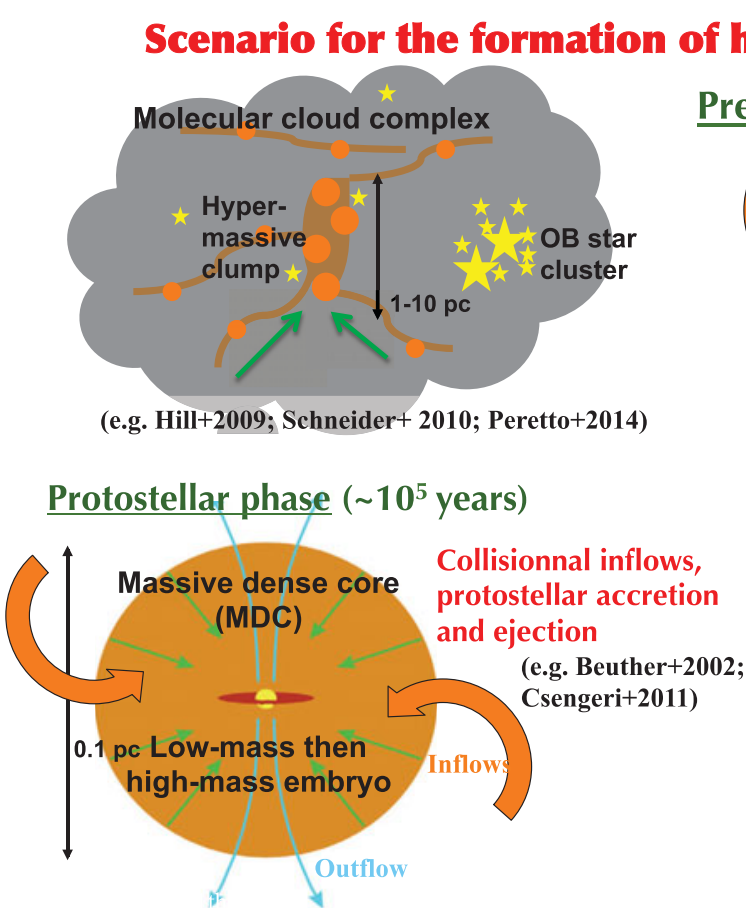

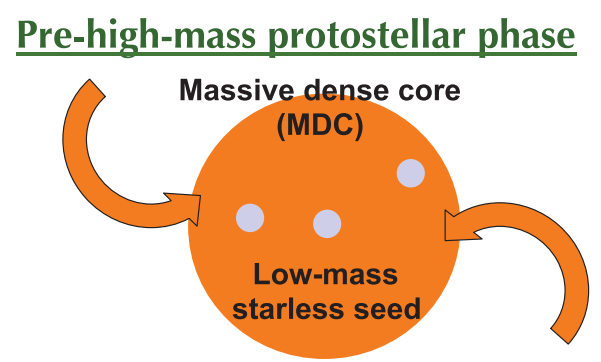

$\underline{\text { HII regions }}\left(\sim 10^{5}-10^{6}\right.$ years $)$

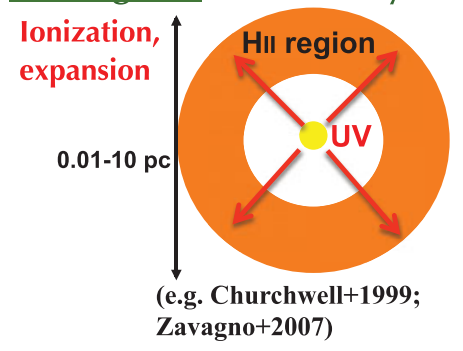

Figure 5. Schematic evolutionary diagram proposed for the simultaneous formation of an hyper-massive filament and one of its high-mass protostars. (A color version of this figure is available online.)

in mass at the same time as hyper-massive clumps will form and get denser. Figure 5 illustrates the evolutionary scheme we propose for the formation of high-mass stars.

In this context, it will be very interesting to investigate, in massive protostellar clusters, the origin of the initial mass function of stars (IMF, e.g., Kroupa 2001). Indeed, while lowmass stars in the Gould Belt clouds accrete their final mass from well-defined prestellar cores with a mass distribution mimicking the IMF (Motte et al. 1998, Könyves et al. 2015), (high-mass) stars within hyper-massive clumps should accrete gas from pc-scales, through sporadic flows, probably favoring the formation of high-mass stars during $10^{5}-$ $10^{6}$ yrs bursts. An ALMA study of the W43-MM1 ridge is ongoing but many more high-resolution studies will be necessary to solve this issue.

\section{References}

André, Ph., Men'shchikov, A., Bontemps, et al. 2010, A\&SA, 518, L102+

Arzoumanian, D., André, Ph., Didelon, P., et al. 2011, A\&3A, 529, L6

Bonnell, I. A. \& Bate, M. R. 2006, MNRAS, 370, 488

Bontemps, S., Motte, F., Csengeri, T., \& Schneider, N. 2010, A\&A, 524, A18

Butler, M. J. \& Tan, J. C. 2012, ApJ, 754, 5

Csengeri, T., Bontemps, S., Schneider, N., Motte, F., \& Dib, S. 2011a, A\& A, 527, A135

Csengeri, T., Bontemps, S., Schneider, N., et al. 2011b, ApJ, 740, L5

Dale, J. E. \& Bonnell, I. 2011, MNRAS, 414, 321

Didelon, P., Motte, F., Tremblin, P. et al. 2015, A\&A, 584, A4

Duarte-Cabral, A., Bontemps, S., Motte, F., et al. 2013, A\&A, 558, A125

Duarte-Cabral, A., Bontemps, S., Motte, F., et al. 2014, A\&GA, 570, A1

Elmegreen, B. 2011, in Star Formation in the Local Universe, EAS Pub. Series, 51, 45 
Federrath, C. \& Klessen, R. S. 2012, ApJ, 761, 156

Federrath, C., Roman-Duval, J., Klessen, R. S., Schmidt, W., \& Mac Low, M.-M. 2010, A\&A, $512, \mathrm{~A} 81$

Galván-Madrid, R., Zhang, Q., Keto, E., et al. 2010, ApJ, 725, 17

Ginsburg, A., Bressert, E., Bally, J., \& Battersby, C. 2012, ApJ, 758, L29

Hartmann, L., Ballesteros-Paredes, J., \& Heitsch, F. 2012, MNRAS, 420, 1457

Heitsch, F. \& Hartmann, L. 2008, ApJ, 689, 290

Hennebelle, P. \& Chabrier, G. 2011, ApJ, 743, L29

Hennemann, M., Motte, F., Schneider, N., et al. 2012, A\&\&A, 543, L3

Henshaw, J. D., Caselli, P., Fontani, F., Jimez-Serra, I., \& Tan, J. C. 2014, MNRAS, 440, 2860

Hill, T., Motte, F., Didelon, P., et al. 2011, A\&SA, 533, A9

Hill, T., Motte, F., Didelon, P., et al. 2012, A\&A, 542, A114

Hosokawa, T. \& Omukai, K. 2009, ApJ, 691, 823

Könyves, V., André, Ph., Men'shchikov, A., et al. 2015, A\&\&A, 584, A91

Kroupa, P. 2001, MNRAS, 322, 231

Krumholz, M. 2014, Physics Reports, 539, Iss. 2, 49-134

Krumholz, M. R. \& McKee, C. F. 2005, ApJ, 630, 250

Louvet, F., Motte, F., Hennebelle, P., et al. 2014, A\& A, 570, A15+

Louvet, F., Motte, F., Gusdorf, A., et al. 2016, soon to be submitted to $A \mathscr{E} A$

McKee, C. F. \& Tan, J. C. 2002, Nature, 416, 59

Menshchikov, A., André, P., Didelon, P., et al. 2012, A\&\&A, 542, A81

Minier, V., Tremblin, P., Hill, T., et al. 2013, A\&SA, 550, A50

Motte, F. \& André, Ph. 2001, A\&A A, 365, 440

Motte, F., André, Ph., \& Neri, R. 1998, A\&̈A, 336, 150

Motte, F., Bontemps, S., Schilke, P., et al. 2007, A\&SA, 476, 1243+

Motte, F., Schilke, P., \& Lis, D. C. 2003, ApJ, 582, 277

Motte, F., Zavagno, A., Bontemps, S., et al. 2010, A\&GA, 518, L77+

Nguyen Luong, Q., Motte, F., Carlhoff, P., et al. 2013, ApJ, 775, 88

Nguyen Luong, Q., Motte, F., Hennemann, M., et al. 2011a, A\&SA, 535, A76

Nguyen Luong, Q., Motte, F., Schuller, F., et al. 2011b, A\&\&A, 529, A41+

Padoan, P. \& Nordlund, A. 2011, ApJ, 730, 40

Peretto, N., Fuller, G. A., Duarte-Cabral, A., et al. 2013, A\&\&A, 555, A112

Russeil, D., Zavagno, A., Motte, F., et al. 2010, A\&A, 515, A55+

Schneider, N., Csengeri, T., Bontemps, S., et al. 2010a, A\& A, 520, A49+

Schneider, N., Csengeri, T., Hennemann, M., et al. 2012, A\& A, 540, L11

Tan, J. C., Kong, S., Butler, M. J., Caselli, P., \& Fontani, F. 2013, ApJ, 779, 96

Tigé, J., Motte, F., Russeil, et al. 2016, soon to be submitted to $A \mathscr{G} A$

Tremblin, P., Audit, E., Minier, V., Schmidt, W., \& Schneider, N. 2012, A\&̛A, 546, A33

Vázquez-Semadeni, E., Kim, J., \& Ballesteros-Paredes, J. 2005, ApJ, 630, L49

Vázquez-Semadeni, E., González, R. F., Ballesteros-Paredes, J., Gazol, A., \& Kim, J. 2008, MNRAS, 390, 769 